\title{
Körperkondition und Stoffwechselstabilität als Grundlage für eine hohe Milchleistung bei ungestörter Fruchtbarkeit und allgemeiner Gesundheit von Milchkühen
}

\begin{abstract}
Title of the paper: Body condition and metabolic stability as basis of high milk yield, reproductive performance, and general health in dairy cows

The target of this study was to describe the interactions between body condition and various descriptors of yield and fertility. It was aimed to identify an optimal conditional range to be used in herd management which combines high milk yield with acceptable fertility traits and general health. For this purpose, backfat thickness was measured by ultrasound at 46111 dairy cows on 78 different farms and was subsequently related to production variables. Negative energy balance is getting more intense and prolonged with increasing milk yield. However a conditional nadir below $10 \mathrm{~mm}$ leads to decreased milk production. To reach a high production level without an increasing incidence of health disorders, conditional nadir should not decline below $13 \mathrm{~mm}$ backfat thickness on herd average. Lower value only lead to negligibly higher milk yield but cause a distinctively higher risk of fertility problems and culling. High herd yields do not have to be at expense of reproduction performance and can be achieved without extreme body condition losses. An efficient herd management can offset depression in fertility, which commonly is combined with increasing milk yield. It is suggested a standard curve for backfat thickness throughout lactation to be used in dairy herd management.
\end{abstract}

Key Words: energy metabolism, body condition, backfat thickness, reference range, milk yield, fertility, dairy cattle

\section{Zusammenfassung}

Zielsetzung der Untersuchungen war die Beschreibung der Wechselwirkungen zwischen der Körperkondition und verschiedenen Milchleistungs- und Fruchtbarkeitsparametern. Es sollten Referenzwerte für einen in der Herdenbetreuung anwendbaren Konditionsbereich ermittelt werden, die hohe Milchleistungen mit akzeptablen Fruchtbarkeitsergebnissen und stabiler Gesundheit vereinen. Dazu wurde bei insgesamt 46111 Kühen in 78 verschiedenen Herden die Rückenfettdicke gemessen und zu Produktionsparametern in Beziehung gesetzt. Mit steigender Milchleistung nehmen Höhe und zeitliche Ausdehnung der negativen Energiebilanz zu. Mittlere Minimalkonditionen unter $10 \mathrm{~mm}$ RFD sind mit Leistungseinbußen verbunden. Um hohe Milchleistungen bei stabiler Gesundheit zu gewährleisten, sollte die Minimalkondition im Herdenmittel bei etwa $13 \mathrm{~mm}$ liegen. Niedrigere Werte steigern die Milchleistung nur unwesentlich, gehen aber mit einer gestörten Fruchtbarkeit und höheren Abgangsraten einher. Hohe Herdenmilchleistungen sind auch ohne extremen Körperkonditionsverlust zu realisieren und schließen gute Fruchtbarkeitsergebnisse nicht aus. Ein leistungsfähiges Management ist in der Lage, der mit steigender Milchleistung tendenziell einhergehenden Fruchtbarkeitsdepression effektiv zu begegnen. Es wird eine Referenzkurve für die Konditionsbeurteilung zur Anwendung in der Bestandsbetreuung vorgeschlagen.

Schlüsselwörter: Energiestoffwechsel, Körperkondition, Rückenfettdicke, Referenzkurve, Milchleistung, Fruchtbarkeit, Milchrind

1. Einleitung

Eine hohe Milchleistung ist obligatorisch an einen intensiven Stoffumsatz gebunden. Dabei kann es bei übermäßiger Favorisierung der Milchsynthese zu Konkurrenzer- 
scheinungen um Energie und Metaboliten mit anderen Organfunktionen kommen, was sich zunächst in Fruchtbarkeitsstörungen und dann im Auftreten von Erkrankungen äußert (STAUFENBIEL u.a., 1991, 1993a, b). Umgekehrt sind solche Kühe, die ihre Körperreserven nur in geringem Maße für die Laktogenese nutzen, weniger krankheitsgefährdet, allerdings auf Kosten einer niedrigeren Milchleistung. Diese Situation lässt sich mit dem Begriff Stoffwechseltyp bzw. Stoffwechselstabilität beschreiben (STAUFENBIEL u.a., 1989a, b, 1992 a).

Unter Stoffwechselstabilität kann der Grad der Beanspruchung der verschiedenen Stoffwechselkreisläufe zur Realisierung einer bestimmten Leistung, im Fall der Kühe der Milchleistung, verstanden werden. Sie ist immer das Ergebnis des Zusammenwirkens von genetischen und umweltabhängigen Faktoren. Bei den Milchkühen wird der Energiestoffwechsel am meisten beansprucht und muss damit im Mittelpunkt der Betrachtung stehen (STAUFENBIEL u.a., 1989b, 1992b, 1993c).

Ein phänotypisches Merkmal, das in direkter Beziehung zum Energiestoffwechsel der Milchkuh steht, ist die Körperkondition, die sich allgemein als Ernährungszustand definieren lässt. Zur Beurteilung der Körperkondition werden verschiedene Methoden eingesetzt. Weit verbreitet ist der durch visuelle Beurteilung ermittelte Body Condition Score (BCS). Der BCS nach der Methode von EDMONSON u.a. (1989) mit einer Benotung zwischen 1 bis 5 hat auf Grund der Einfachheit weite Verbreitung im Herdenmanagement gefunden. Allerdings stellt der BCS immer eine subjektive Methode dar, die nur eine grobe Differenzierung zulässt. Im Unterschied dazu ist die mit Ultraschall gemessene Fettauflage an einem definiertem Punkt im Rückenbereich ein objektives Maß für die Quantität der vorhandenen Körperfettreserven (STAUFENBIEL, 1992, 1997). Zwischen der Rückenfettdicke (RFD) und dem Gesamtkörperfettgehalt besteht bei Milchkühen eine phänotypische Korrelation um 0,8. $1 \mathrm{~mm}$ gemessener RFD entsprechen ca. 5 kg Körperfett (KLAWUHN und STAUFENBIEL, 1997a, b). Die laktationsabhängigen Veränderungen zwischen Katabolismus und Anabolismus werden durch die RFD anschaulich widergespiegelt. Die Abnahme der RFD ist ein quantitatives Maß für die Lipolyseintensität, die Zunahme für die Lipogeneserate (STAUFENBIEL, 1997) bzw. für die Energiebilanz (STAUFENBIEL u.a., 1993b, c).

In den nachfolgend dargestellten Untersuchungen soll der Frage nachgegangen werden, inwiefern das Ultraschallmaß der Rückenfettdicke (RFD) zur Charakterisierung der Wechselbeziehung zwischen Milchleistung und Tiergesundheit beitragen kann. Wünschenswert wäre das Herausarbeiten einer Methode zur Abgrenzung des Bereiches zwischen synergistischer (gewünschter) und antagonistischer (unerwünscht) Beziehung zwischen Milchleistung und Tiergesundheit. Diese Aufgabenstellung impliziert die immer wieder diskutierte Frage nach der Beziehung zwischen der Höhe der Milchleistung und der Tiergesundheit, oder anders formuliert, sind Kühe mit einer hohen Milchleistung anfälliger gegenüber Erkrankungen.

2. Material und Methoden

Tiermaterial

In einem Gesamtuntersuchungszeitraum von einem Jahr wurde in 78 Betrieben in den Ländern Brandenburg, Mecklenburg-Vorpommern und Sachsen-Anhalt bei insgesamt 46111 Kühen die Rückenfettdicke gemessen. 10 Betriebe wurden zur Erfassung der Änderung der Rückenfettdicke im Abstand von 4 Wochen wiederholt berücksichtigt. Dadurch verteilen sich die Messungen auf insgesamt 108 Betriebsbesuche. Die mitt- 
lere Herdengröße lag bei 400 Kühen bei einer Variation zwischen 50 und 3500. Pro Herde wurden 46 bis 1165 Kühe, im Durchschnitt 400 Kühe untersucht. Die Auswahl der Probanden erfolgte zufällig, so dass von einer repräsentativen Stichprobe ausgegangen werden kann. Die mittlere 305-Tagemilchleistung bewegte sich in den Herden zwischen 5800 und 10200 kg Milch. In der Mehrzahl der Fälle wurde zweimal gemolken. In der Fütterung dominierte die TMR. Die Haltung erfolgte ausschließlich im Laufstall in Kombination mit einer kurzzeitigen Anbindehaltung von trockenstehenden und frischabgekalbten Kühen. Mit 87\% gehörte die Mehrzahl der untersuchten Kühe einer Verdrängungskreuzung aus Schwarzbuntem Milchrind (SMR) mit Holstein-Frisian(HF)-Insemination an. Der HF-Anteil schwankte zwischen 75 und 90 \%. Daneben ließen sich 8\% der Probanden eindeutig der Rasse HF zuordnen. Die restlichen Tiere gehörten zur Rasse des Deutschen Rotbunten Rindes oder zu verschiedenen Fleischrindkreuzungen.

\section{Untersuchungsmethoden}

Die Messung der Rückenfettdicke erfolgte entsprechend den Angaben von STAUFENBIEL (1992, 1997) mit einem akkubetriebenen, transportablen Ultraschallgerät. Als Schallkopf diente ein 5-MHz-Linearscanner, der direkt nach Anfeuchten von Haut und Haarkleid unter Verwendung von verdünntem Alkohol auf den Messpunkt im Kruppenbereich eine Handbreite vor der Schwanzfaltengrube aufgelegt wurde. Der Rückenfettdicke(RFD)-Messwert enthält die Hautdicke und wird in mm angegeben. Da die Haut relativ konstant um $6 \mathrm{~mm}$ beträgt, entspricht dies dem geringsten Messwert für die RFD. Der Messfehler lag um 5\% bei einer maximalen Abweichung bis zu 2,3 mm bei Einzeltieren. Der eigentliche Messvorgang dauerte nur Sekunden. Unter Berücksichtigung der notwendigen Tierfixierung betrug der Zeitaufwand 30 bis 60 Minuten pro 100 Tiere in Abhängigkeit von der Herdengröße und der Aufstallungsform.

\section{Datenerfassung}

Von jeder Kuh wurde zum Untersuchungszeitpunkt die betriebseigene Identifizierungsnummer erfasst. Die Mehrzahl der Betriebe verfügte über das Herdenverwaltungsprogramm Herde2 ${ }^{\circledR}$ (dsp-Agrosoft, Paretz), ein anderer Teil über Superkuh III ${ }^{\circledR}$ (Klöpper \& Wiege, Lemgo). Von den verbleibenden 20\% an Betrieben ohne eines dieser kommerziellen Herdenverwaltungsprogramme wurden die Herdendaten direkt aus dem Zentralrechner des VIT Paretz entnommen. In Zusammenarbeit mit der dspAgrosoft wurde ein Programmmodul entwickelt, um die verschiedenen Datenformate zu vereinheitlichen, um dann über das Programm Zuchtmanager ${ }^{\circledR}$ der dsp-Agrosoft die erforderlichen Auswertungen durchführen und die Ergebnisse zur weiteren statistischen Auswertung in das Programm MS Excel 7.0 ${ }^{\circledR}$ und SPSS 8.0 Windows ${ }^{\circledR}$ übernehmen zu können. Diese umfangreichen Vorarbeiten sicherten die Bereitstellung der zur Auswertung notwendigen Daten. Zunächst wurde neben der Ohrnummer die Laktationszahl, der Laktationstag zum Zeitpunkt der Messung sowie die Rasse zugeordnet. Auf Basis der monatlichen Milchleistungskontrolldaten wurden die Milchmenge in kg und kg FCM (fat corrected milk), der Milchfettgehalt in \%, der Milchproteingehalt in $\%$, der Laktosegehalt in \%, der Harnstoffgehalt in $\mathrm{mg} / \mathrm{l}$ sowie die Zellzahlen in 1000/ml erfasst. Ausgewertet wurde das Ergebnis der zum Messzeitpunkt aktuellen 
Milchleistung, die Einsatzleistung, die hochgerechneten 100- und 305-Tageleistungen. Um die Vergleichbarkeit der Milchleistung zwischen den verschiedenen Herden herzustellen, wurde ein sogenannter relativer Leistungswert (RLW) kalkuliert. Dazu wurde in jeder Herde der Mittelwert für die 305-Tageleistung, getrennt für die Kühe der ersten, zweiten und ab der dritten Laktation, berechnet. Zu diesem Wert wurde durch Subtraktion die tierindividuelle Leistung in Beziehung gesetzt. Der RLW gibt damit an, wieviel kg Milch eine Kuh mehr oder weniger in Relation zum laktationszahlbezogenen Herdendurchschnitt ermolken hat. Zur Bewertung der Fruchtbarkeit wurden die Rastzeit, die Zwischentragezeit, die Verzögerungszeit, die Zwischenkalbezeit und der Besamungsindex einbezogen. Problematisch war die Datenerfassung zur Tiergesundheit. Hier konnten nur das Abgangsdatum und Abgangsursache eindeutig zugeordnet werden. Von den insgesamt 46111 ließen sich 43506 auswertbare Datensätze von Kühen aus 75 Betrieben mit insgesamt 105 Besuchen zusammenstellen. Diese Stichprobe wurde vor der statistischen Auswertung weiter reduziert. Ausgeschlossen wurden Kühe mit einer Trockenstehzeit über 150 Tagen, mit einer Laktationsdauer über 600 Melktagen sowie solche Kühe, die sich nicht der Kreuzung SMRxHF oder der Rasse HF zuordnen ließen. Dadurch reduzierte sich die Stichprobenzahl weiter auf 36019 Kühe (Tab. 1).

\section{Statistische Auswertung}

Die statistische Auswertung erfolgte mit Hilfe des Statistikprogramms SPSS 8.0 Windows $^{\circledR}$ (Microsoft Corporation). Zunächst wurden zur Beschreibung der Werteverteilung die üblichen statistischen Maßzahlen berechnet. Zur Darstellung der Beziehung zwischen den verschiedenen Leistungsparametern (Milchmengenleistung, Milchproteingehalt, Zwischentragezeit) und dem Verlauf der Rückenfettdicke im Laktationszyklus wurden die Probanden nach den entsprechenden Parametern gruppiert und dann mit Hilfe einer Kovarianzanalyse nach der General Linear Model (GLM)-Prozedur ausgewertet. Die RFD wurde als abhängig Variable von dem jeweils ausgewählten Leistungsparameter betrachtet. Der Laktationstag wurde bis zur vierten Potenz als Kovariate in die Berechnung einbezogen. Der aus der Berechnung resultierende p-Wert beschreibt den Gruppeneffekt für den ausgewerteten Leistungsparameter. Das Signifikanzniveau wurde mit $p=0,05$ festgelegt. Weiterhin wird das Bestimmtheitsmaß $R^{2}$ angeführt. Es beschreibt den Anteil der Variation, der durch das Modell erklärt wird. Zur Beschreibung der Verlaufskurve der gemessenen RFD-Werte über den Untersuchungszeitraum wurde eine polynomiale Regression unter Anwendung der Prozedur Linear Regression aus SPSS 8.0 Windows ${ }^{\circledR}$ eingesetzt. Die RFD ist die abhängige Variable. Die Melktage wurden als unabhängige Variable bis zur vierten Potenz berücksichtigt. Die Auswertung erfolgte getrennt für den Zeitraum der Trockenstehperiode und für die Laktationsperiode. Diese Regressionsanalyse wurde zur Modellierung der Referenzkurve sowie zur Darstellung der Wirkung verschiedener Effekt auf den Verlauf der RFD im Laktationszyklus herangezogen.

\section{3. $\quad$ Ergebnisse}

Tabelle 1 gibt einen Überblick zu den auswertbaren Stichprobenzahlen, zum Wertebereich, den Mittel- und Medianwerten für die in die Untersuchung einbezogenen Untersuchungsgrößen. Mittelwerte und Medianwerte liegen überwiegend eng beieinander, 
was auf eine relativ gute Anpassung an eine Normalverteilung hinweist. Für die relative Leistung RLW liegen sie um den Nullpunkt, was die Richtigkeit der Kalkulation bestätigt. Bemerkenswert ist die Variationsbreite für alle Parameter. Die gemessenen Rückenfettdicken variieren zwischen 6 und 60 mm (Tab. 1). Die Spannweite der RLW weist aus, dass innerhalb der Herden die schlechtesten Kühe bis 7051 kg unterhalb und die besten bis $9458 \mathrm{~kg}$ oberhalb der mittleren 305-Tage-Herdenmilchmengenleistung liegen (Tab. 1). In ähnlicher Weise variieren auch die anderen Untersuchungsgrößen über einen ausgedehnten Bereich (Tab. 1). Damit liegen gute Voraussetzungen für die statistische Auswertung zur Beschreibung der Wechselbeziehungen zwischen den Leistungsparametern und der RFD vor.

Tabelle 1

Mittelwert ( $\bar{x})$, Standardabweichung (s), Median ( $\left.\mathrm{x}_{0,5}\right)$, Minimum und Maximum ausgewählter Fruchtbarkeitsund Milchleistungsdaten (Data list of some parameters of fertility performance and milk yield)

\begin{tabular}{|c|c|c|c|c|c|c|}
\hline Merkmal & $\mathrm{n}$ & $\bar{x}$ & $\mathrm{~s}$ & $\mathrm{x}_{0,5}$ & Minimum & Maximum \\
\hline$\overline{R F D}(\mathrm{~mm})$ & 36019 & 17,9 & 7,68 & 17 & 6 & 60 \\
\hline Laktationszahl & 36019 & 2,5 & 1,6 & 2 & 1 & 12 \\
\hline Besamungsindex & 26018 & 2,0 & 1,3 & 2 & 1 & 14 \\
\hline RZ (Tage) & 30965 & 85,1 & 40,2 & 76 & 20 & 654 \\
\hline VZ (Tage) & 25805 & 41,1 & 61,9 & 15 & 0 & 706 \\
\hline ZTZ (Tage) & 26792 & 124,4 & 70,0 & 105 & 20 & 836 \\
\hline ZKZ (Tage) & 26752 & 400,1 & 68,4 & 381 & 300 & 795 \\
\hline HR100M (kg) & 23187 & 3028 & 759 & 3003 & 109 & 6233 \\
\hline HR100F (kg) & 23189 & 122,0 & 31,6 & 120,3 & 24,1 & 370,2 \\
\hline HR100E (kg) & 23188 & 98,0 & 23,5 & 97,4 & 20,5 & 184,3 \\
\hline HR305M (kg) & 28460 & 7759 & 1824 & 7642 & 1616 & 16310 \\
\hline HR305F (kg) & 28135 & 323,3 & 72,1 & 319,2 & 4,2 & 684,0 \\
\hline HR305E (kg) & 28046 & 267,2 & 57,6 & 265 & 61,0 & 494,0 \\
\hline HR305FCM (kg) & 28039 & 7945 & 1729 & 7872 & 1831 & 14767 \\
\hline RLW (kg) & 28460 & $-0,56$ & 1396 & -8 & -7051 & 9458 \\
\hline HR305F\% (kg) & 28039 & 4,23 & 4,21 & 0,53 & 0,03 & 7,45 \\
\hline HR305E\% (kg) & 28046 & 3,48 & 3,47 & 0,25 & 2,46 & 4,97 \\
\hline
\end{tabular}

$\bar{x} \pm \mathrm{s} \quad$ Mittelwert \pm Standardabweichung $; \mathrm{x}_{0,5}$ Medianwert

Die Entwicklung der RFD im Laktationsverlauf zeigt eine hochsignifikante Differenzierung zwischen Kühen mit unterschiedlicher Milchleistung. Dieser Zusammenhang wurde für die Gruppierung nach dem Leistungsniveau der Herde, nach der individuellen Milchleistung der Kuh unabhängig von der Herde sowohl für die Original- als auch FCM-Leistung nachgewiesen. Am deutlichsten stellt sich dieser Zusammenhang allerdings zur relativen Milchleistung dar (Abb. 1). Mit steigender Milchleistung werden die Körperfettdepots intensiver und länger mobilisiert. Auch der Wiederaufbau der Fettdepots bis zum Ende der Laktation zeigt eine nahezu parallele Verlaufskurve bei einem fallenden Niveau mit steigender Milchleistung. Allerdings gleichen sich die Unterschiede in der Trockenstehperiode an. Dies vollzieht sich in dem in der Abbildung 1 nicht mehr dargestellten Zeitraum nach dem 340. Laktationstag. Das lässt zugleich den Schluss einer längeren Laktationsdauer bei hoher relativer Milchleistung zu.

Von den Milchinhaltsstoffen erbringt die Gruppierung nach dem Milchproteingehalt die deutlichste Differenzierung zum Laktationsverlauf der RFD (Abb. 2). Zwar besteht auch zur absoluten Milchproteinmenge sowie zum Milchfettgehalt und zur Milchfettmenge eine hochsignifikante Beziehung, die allerdings in ihrer Ausprägung deutlich hinter der des Milchproteingehaltes zurücksteht. Zunächst fällt auch bei der Gruppie- 
rung nach dem Milchproteingehalt auf, dass in der Trockenstehperiode keine Unterschiede bestehen. Im Laktationszyklus stellt sich der Verlauf der RFD wie parallel verschobene Linien dar. Ein niedriger mittlerer 305-Tage-Milchproteingehalt geht mit einer längeren und intensiveren Fettmobilisation und einer verzögerten Fettdeposition einher. Wie bei der Milchmengenleistung muss bei den Kühen mit niedrigerem Milchproteingehalt nach dem in Abbildung 2 dargestellten Zeitraum von 340 Laktationstagen ein wesentlicher Fettansatz stattfinden, um das in der Trockenstehperiode gleiche Niveau zu erzielen.

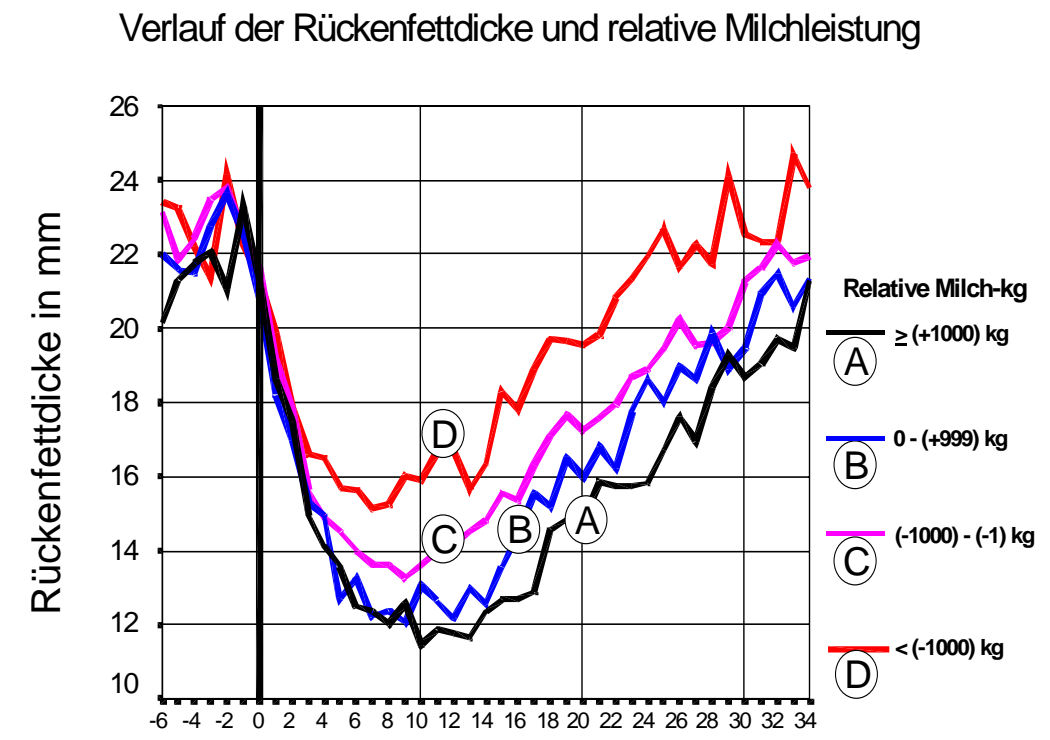

Tage post partum $\times 10$

Abb. 1: Verlaufskurven der Rückenfettdicke in Zuordnung zur relativen Milchleistung der Kühe auf Herdenebene ( $p<0,001$ für den Gruppeneffekt, $R^{2}=0,194$ ) (Backfat thickness curve in relation to relative milk yield of individual cow on herd level. $\mathrm{p}<0.001$ for group effect, $\mathrm{R}^{2}=0.194$ )

\section{Verlauf der Rückenfettdicke und Milchproteingehalt}

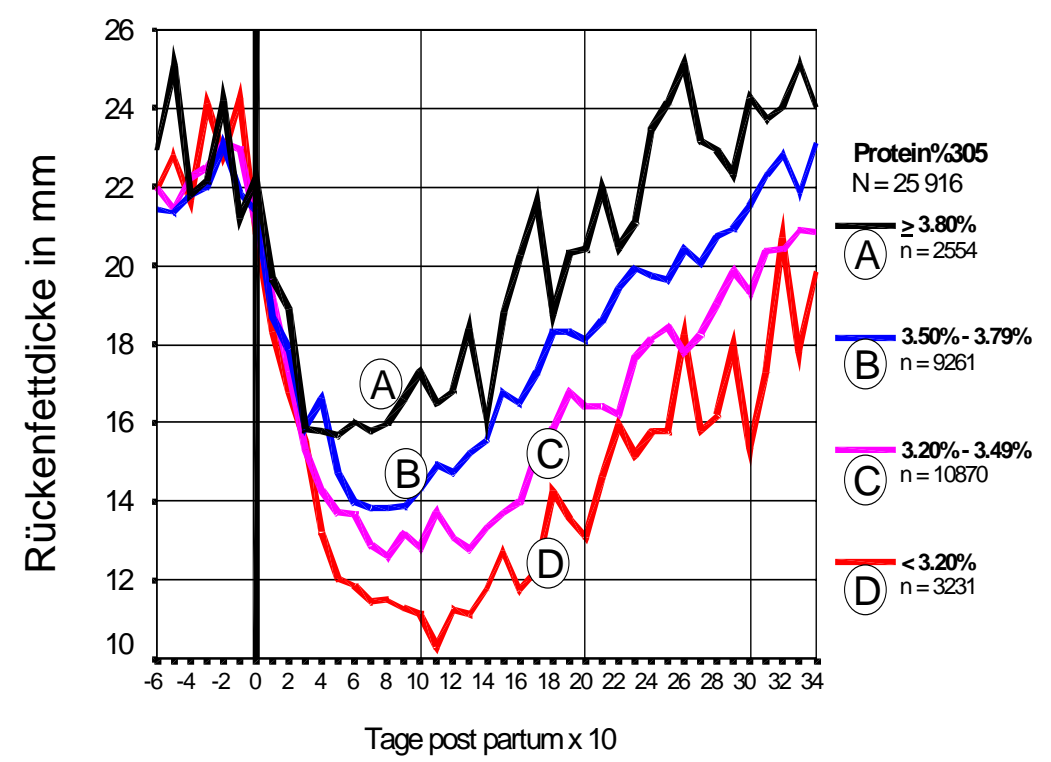

Abb. 2: Verlaufskurven der Rückenfettdicke in Zuordnung zum Milchproteingehalt. ( $<<0,001$ für den Gruppeneffekt, $\mathrm{R}^{2}=0,191$ ) (Backfat thickness curve in relation to milk protein content. $\mathrm{p}<0.001$ for group effect, $\left.\mathrm{R}^{2}=0.191\right)$ ) 


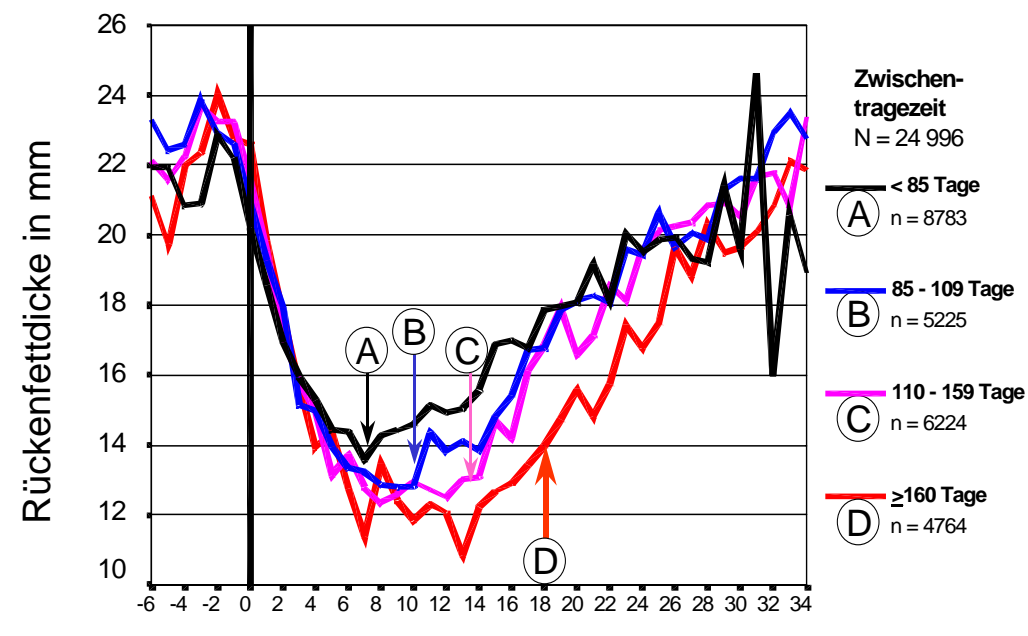

Tage post partum $\times 10$

Abb. 3: Verlaufskurven der Rückenfettdicke in Zuordnung zur Zwischentragezeit. ( $p<0,001$ für den Gruppeneffekt, $\mathrm{R}^{2}=0,166$ ) (Backfat thickness in relation to calving conception interval. $\mathrm{p}<0.001$ for group effect, $\mathrm{R}^{2}=0.166$ )

Von den Fruchtbarkeitsparametern lassen sich für die Rastzeit, Zwischentragezeit, Verzögerungszeit und Zwischenkalbezeit signifikante Einflüsse auf den Verlauf der RFD nachweisen. Am interessantesten ist die Gruppierung nach der Zwischentragezeit (Abb. 3). Eine zunehmende Zwischentragezeit ist mit einem signifikant intensiveren und längeren Abbau bzw. verzögertem Wiederaufbau der Körperfettreserven verbunden. In Abbildung 3 wurde der mittlere Zeitpunkt der Konzeption in den 4 Gruppen markiert. In den Gruppen mit einer Zwischentragezeit unter 85 Tagen, von 85 bis 109 Tagen und 110 bis 159 Tagen werden die Kühe im Durchschnitt in zeitlicher Nähe des Wendepunktes der RFD und damit des Wechsels vom Fettabbau zum Fettaufbau tragend. Nur bei den Kühen mit einer Zwischentragezeit über 159 Tagen tritt die Trächtigkeit wesentlich später ein.

\section{Tabelle 2}

Angaben zur Regressionsgleichung zur Beschreibung der Referenzkurve in Abbildung 4 (Parameter of regression equation for the backfat standard curve in picture 4)

Laktationsperiode

\begin{tabular}{c|c|c}
\multicolumn{2}{c}{ RFD $=22,069-0,236 \mathrm{~d}+2,01 * 10^{-3} \mathrm{~d}^{2}-6,0 * 10^{-6} \mathrm{~d}^{3}+6,32 * 10^{-9} \mathrm{~d}^{4}$} \\
\hline $\mathrm{n}=29106$ & S.E. $=6,60$ & $\mathrm{R}^{2}=0,146$ \\
\hline $\begin{array}{c}\text { Trockenstehperiode } \\
\text { RFD }=22,987-3,2 * 10^{-2} \mathrm{~d}-1,7 * 10^{-3} \mathrm{~d}^{2}+3,32 * 10^{-7} \mathrm{~d}^{4}\end{array}$ \\
\hline $\mathrm{n}=4306$ & S.E. $=7,49$ & $\mathrm{R}^{2}=0,002$ \\
\hline
\end{tabular}

d Laktationstag

Auch für Gruppierung nach der Laktationszahl, nach der Saison (Jahreszeit) und nach der Rasse können signifikante Beziehungen zum Verlauf der RFD nachgewiesen werden. So nimmt erwartungsgemäß mit steigender Laktationszahl der Fettansatz im Laktationsverlauf signifikant zu. Reinrassige HF-Kühe nutzen die Fettreserven intensiver als die Kreuzungstiere SMRxHF. Allerdings muss beachtet werden, dass erstere auch eine signifikant höhere Milchleistung aufwiesen.

Da sich für relevante Kennziffern der Milchleistung und Fruchtbarkeit signifikante Beziehungen zum Verlauf der RFD in der Laktation nachweisen ließen, ergibt sich die 
Frage nach dem optimalen Verlauf der RFD und damit nach einer Referenzkurve. Zu diesem Zweck wurden die verfügbaren Messwerte zur Kalkulation eines polynomialen Regressionsmodelles herangezogen (Abb. 4). Die Auswertung erfolgte getrennt für die Trockenstehperiode und Laktation. Die Regressionsgleichungen sind der Tabelle $2 \mathrm{zu}$ entnehmen. Die Abbildung 4 beschreibt den anzustrebenden Konditionsverlauf als Mittelwert als auch den dazugehörigen Streuungsbereich für die Einzeltiere.

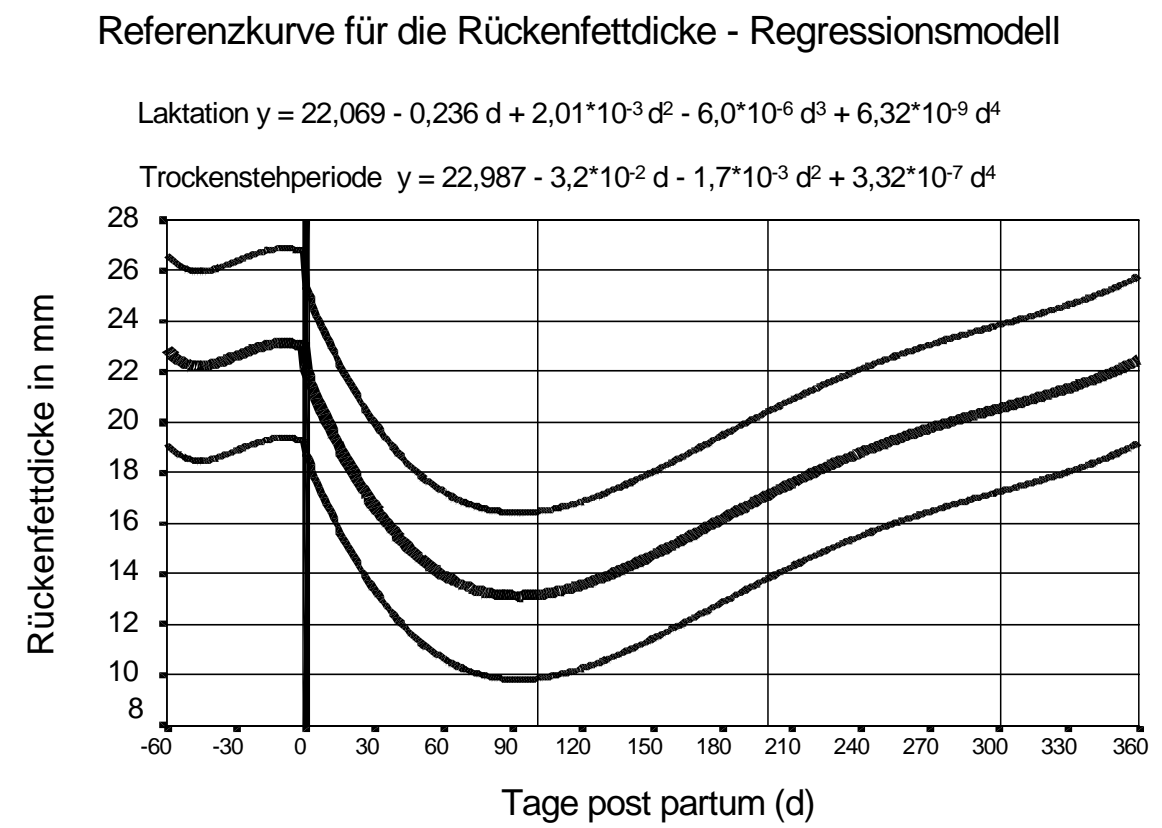

Abb. 4: Referenzkurve für die Rückenfettdicke auf der Basis einer polynomialen Regressionsfunktion (Vergleiche Tab. 2) (Standard curve for backfat thickness on basis of a polinomial regression function. See table 2)

Verlauf der Rückenfettdicke in Betrieb 1

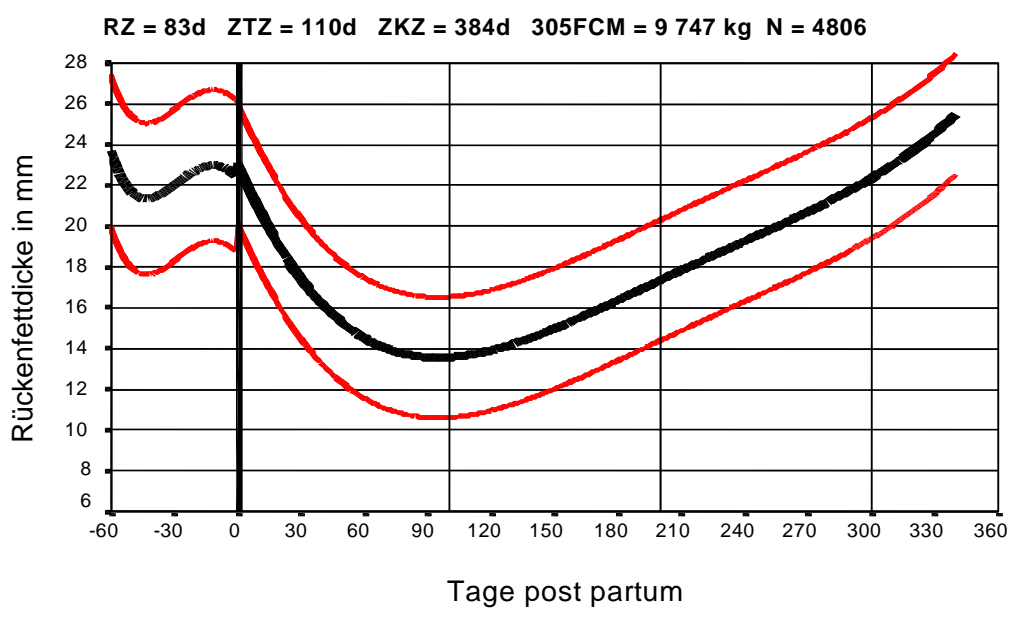

Abb. 5: Rückenfettdicke, Milchleistung und Fruchtbarkeitsergebnisse in Herde 1 (Backfat thickness, yield and fertility traits in herd 1)

Die Relevanz dieser Referenzkurve soll an zwei größeren Herden dargestellt werden. In diesen Herden standen eine ausreichende Zahl an Einzelmesswerten zur Verfügung, um sie mit dem beschriebenen Regressionsmodell auszuwerten (Abb. 5, 6). Betrieb 1 erreicht mit einer mittleren 305-Tageleistung von 9747 kg FCM eine akzeptable 
Milchleistung. Auch die Fruchtbarkeitskennzahlen sind mit einer Rastzeit von 83 Tagen und einer Zwischentragezeit von 110 Tagen für das Leistungsniveau ansprechend. Die Milchleistung in Betrieb 2 erreicht mit 9366 kg ein vergleichbares Niveau. Allerdings sind die Fruchtbarkeitsergebnisse mit einer Rastzeit von 102 Tagen und einer Zwischentragezeit von 140 Tagen unbefriedigend. Vergleicht man nun die Verlaufskurven für die RFD, sind sie für die Trockenstehperiode gleich, unterscheiden sich jedoch in der Laktation. In Betrieb 1 liegt der Tiefpunkt der RFD zwischen 13 und 14 mm, der Wendepunkt zwischen 90 und 100 Tagen post partum (Abb. 5). Damit entspricht der Verlauf der RFD nahezu der Referenzkurve (Abb. 4). Anders verhält es sich in Betrieb 2. Die Fettreserven werden weitaus stärker ausgenutzt, als es in der Referenzkurve ausgewiesen wird (Abb. 6, 4). Das lässt vermuten, dass in Betrieb 2 im Unterschied zu Betrieb 1 die Milchleistung in Konkurrenz zur Fruchtbarkeit getreten ist.

Verlauf der Rückenfettdicke in Betrieb 2

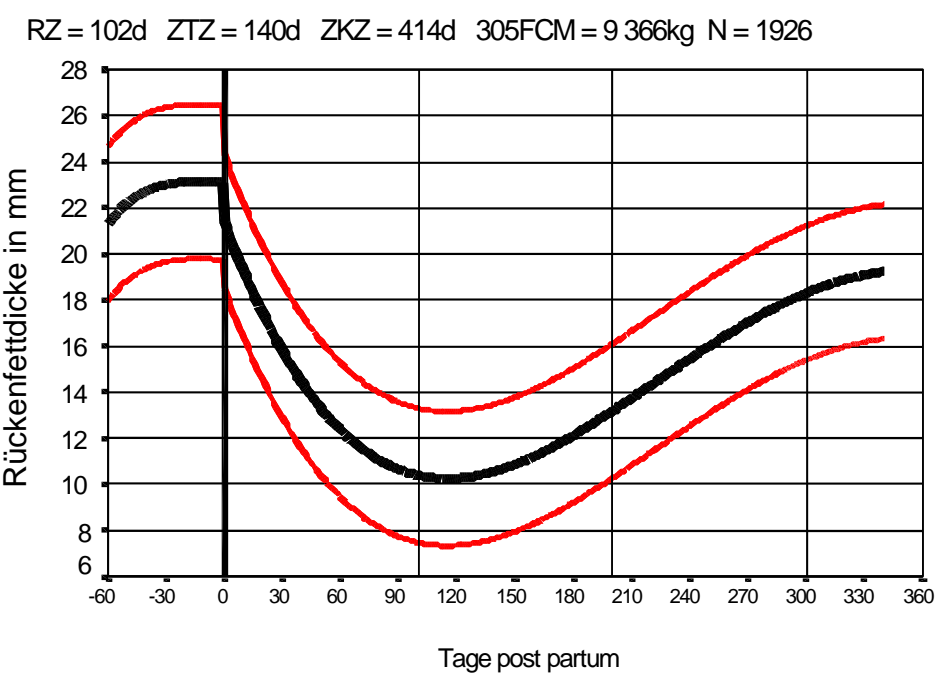

Abb. 6: Rückenfettdicke, Milchleistung und Fruchtbarkeitsergebnisse in Herde 2 (Backfat thickness, yield and fertility traits in herd 2)

Um dieser Überlegung nachzugehen, wurde das verfügbare Datenmaterial mit Hilfe der Regressionsanalyse in drei Gruppen ausgewertet. Die Kühe in Gruppe A wurden ausschließlich nach dem Kriterium einer sehr guten Fruchtbarkeit (Zwischenkalbezeit kleiner 366 Tage) ausgewählt. Die Tiere in Gruppe B sollen sowohl eine gute Fruchtbarkeit (Zwischenkalbzeit kleiner 381 Tage) und eine hohe Milchleistung (305-Tageleistung über $9000 \mathrm{~kg}$ FCM) aufweisen. Gruppe C wurde hingegen ausschließlich nach einer sehr hohen Milchleistung (305-Tageleistung über 9500 kg FCM) zusammengestellt. Abbildung 7 bringt anschaulich zum Ausdruck, dass die einseitige Auswahl nach guten Fruchtbarkeitsergebnissen mit einer geringeren Nutzung der Körperfettreserven einhergeht, wie umgekehrt die einseitige Auswahl ausschließlich nach der Milchleistung mit einer intensiveren Fettmobilisation verbunden ist (Abb. 7). Wird hingegen sowohl auf eine hohe Milchleistung und eine gute Fruchtbarkeit Wert gelegt, dann verläuft die Kurve für die RFD nicht nur zwischen den erstgenannten, sondern entspricht in ihrem Verlauf wieder nahezu der Referenzkurve (Abb. 4). Das ist als deutlicher Beleg in Verbindung mit den Ergebnissen aus Abbildungen 5 und 6 zu bewerten, dass die Referenzkurve aus Abbildung 4 tatsächlich auch als Optimalkurve für den Konditionsverlauf auf Basis der RFD-Messung anzusehen ist, wenn man als Ziel- 
stellung eine harmonische Entwicklung von Leistung und Fruchtbarkeit voraussetzt und umgekehrt eine einseitige Orientierung nur an der Milchleistung vermeiden will.

Rückenfettdicke bei differenzierter Leistung und Fruchtbarkeit

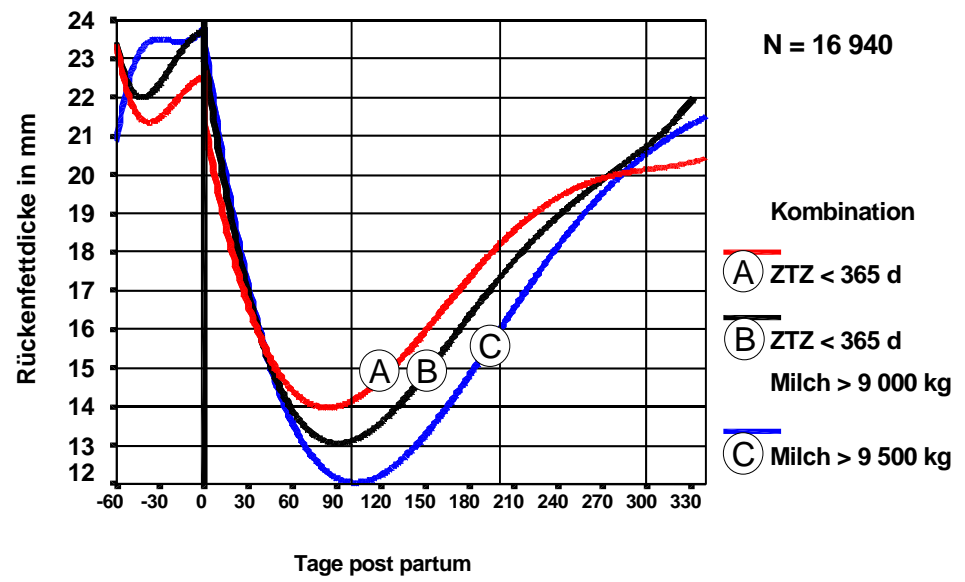

Abb. 7: Verlaufskurven der Rückenfettdicke in Zuordnung zu den Fruchtbarkeitsergebnissen, zur Milchleistung und zu einer Kombination aus Fruchtbarkeit und Milchleistung (Backfat thickness in relation to fertility traits, milk yield or both of them)

\section{Diskussion}

In den vorgestellten Untersuchungen konnte ein enger Zusammenhang zwischen der Milchleistung und der Rückenfettdicke nachgewiesen werden (Abb. 1). Das steht in guter Übereinstimmung mit eigenen früheren Untersuchungen (STAUFENBIEL u.a., 1991, 1992b; STAUFENBIEL, 1997) wie auch mit Ergebnissen der Literatur, die allerdings als Konditionsmaß den BCS nutzten (WILDMAN u.a., 1982; VEERKAMP u.a., 1994). Gut ausgebildete Fettdepots zum Abkalbezeitpunkt wirken über ihre metabolische und energetische Nutzung fördernd auf die Milchleistung (PEDRON u.a., 1993; MARKUSFELD u.a., 1997; DOMECQ u.a., 1997a). Eine Überkonditionierung bedingt jedoch umgekehrt Milchleistungseinbußen in Verbindung mit einem erhöhten Risiko für das Auftreten verschiedener Erkrankungen (GEARHART u.a., 1990; WALTNER u.a., 1993; STAUFENBIEL, 1997). In verschiedenen Untersuchungen wurde nachgewiesen, dass eine steigende Milchleistung mit einem stärkeren Konditionsverlust bei zugleich späterem Eintritt des Wechsels von der negativen zur positiven Energiebilanz verbunden ist (PEDRON u.a., 1993; RUEGG und MILTON, 1995; GALLO u.a., 1996). So leicht verständlich diese Aussage zunächst sein mag, wirft sie jedoch ein grundsätzliches Problem auf. Irgendwann müssten die Kühe an einen Punkt kommen, an dem sämtliche Konditionsreserven aufgebraucht sind. Auch müssten Hochleistungskühe grundsätzlich schlechter als Kühe mit geringerer Leistung konditioniert sein. Das widerspricht der Erfahrung und dem Verständnis für physiologische Zusammenhänge. Aber genau an dieser Stelle setzt der große Wert und die neue Qualität der vorgestellten Untersuchungen an.

Die herauszustellende Besonderheit liegt im Umfang des Tiermaterials unter Berücksichtigung einer großen Zahl verschiedener Herden. Die Nutzung von Herdenmanagementprogrammen ermöglicht die Auswertung verschiedener Einzeltierdaten. Die festgestellte Variationsbreite der erfassten Parameter ist im Sinne der Zielstellung als positiv herauszustellen (Tab. 1). 
Die Differenzierung der RFD ist bei der Auswertung der relativen Milchleistung am größten (Abb. 1). Das bedeutet, dass sich die Wechselbeziehungen zwischen Milchleistung und Körperkondition am stärksten zwischen Kühen innerhalb einer Herde unter gleichen Umweltbedingungen ausprägen. Offensichtlich entwickelt sich eine annähernd gleiche Differenzierung der RFD auf einem unterschiedlichen Milchleistungsniveau. In Übereinstimmung mit dieser Aussage wird der Zusammenhang zwischen Milchleistung und RFD beim direkten, herdenübergreifenden Vergleich schlechter. Wird der Verlauf der RFD in Abhängigkeit von der Leistungsdifferenzierung zwischen den Herden verglichen, liegen die Kurven für die RFD eng beieinander. Daraus leitet sich die einfache, aber grundsätzliche und wichtige Schlussfolgerung ab, dass sich die als optimal zu betrachtende Kondition leistungsunabhängig auf dem individuellen Leistungsniveau einer Herde immer wieder neu einstellt. Der milchleistungsfördernde Effekt der Körperenergiedepots manifestiert sich in erster Linie innerhalb einer Herde und hier wiederum relativ unabhängig von deren absoluten Milchleistungsniveau.

Das erklärt auch die deutlich bessere Differenzierung der Verlaufskurven der RFD für den Milchproteingehalt im Vergleich zur absoluten Milchproteinmenge (Abb. 2). Die absolute Milchproteinmenge wird wesentlich von der Milchmengenleistung bestimmt. Der Milchproteingehalt ist hingegen zunächst einmal relativ unabhängig von der Milchmengenleistung und steht der ausgewerteten relativen Milchmengenleistung näher. Unerwartet ist, dass der Milchproteingehalt eine engere Beziehung zur RFD als der Milchfettgehalt aufweist. Der Abbau der Körperkondition geht sowohl mit der Bereitstellung von Metaboliten für die Milchfettsynthese als auch für die Energiebildung einher. Der Milchproteingehalt gilt als empfindlicher Parameter für die Energieversorgung, wobei ein Abfall des Milchproteingehaltes eine energetische Unterversorgung anzeigt (LOTTHAMMER, 1981; HEUER u.a., 1999). Ein Milchproteingehalt unter $3,2 \%$ wird als Hinweis auf eine deutliche energetische Unterversorgung angesehen. In Abbildung 2 wird die RFD bei den Kühen mit einem mittleren Milchproteingehalt unter 3,2\% am längsten und am intensivsten abgebaut, so dass in Übereinstimmung mit der Bewertung des Milchproteingehaltes von einer übermäßigen, gesundheitsgefährdenden Ausprägung des postpartalen Energiedefizits gesprochen werden muss. Im Unterschied dazu nutzen die Kühe mit einem mittleren Milchproteingehalt von über 3,8\% ihr Milchleistungsvermögen nicht aus, was mit einer geringen Nutzung der Körperkondition verbunden ist (Abb. 2).

Eine intensive Ausprägung der negativen Energiebilanz in der Frühlaktation mit einer stärkeren Mobilisation von Körperfett ist mit schlechteren Fruchtbarkeitsergebnissen verbunden (STAPLES u.a., 1990; STAUFENBIEL u.a., 1991; NEBEL und Mc GILLIARD, 1993; MARKUSFELD u.a., 1997; DOMECQ u.a., 1997b). In guter Übereinstimmung damit stehen die Ergebnisse aus Abbildung 3, die ebenfalls auf einen engen Zusammenhang zwischen der postpartalen Konditionsentwicklung und dem Fruchtbarkeitsgeschehen hinweisen. Bemerkenswert ist der enge Zusammenhang zwischen dem Tiefpunkt der Konditionsentwicklung und der erfolgreichen Konzeption, der sich für drei der vier Gruppen darstellt (Abb. 3). Das lässt den Schluss zu, dass bei der Mehrzahl der ausgewerteten Kühe das Fruchtbarkeitsgeschehen wesentlich von der Konditionsentwicklung beeinflusst wird. Nur in der Gruppe mit der längsten Zwischentragezeit wird dieser Zusammenhang aufgehoben, was auf andere, von der Kondition unabhängige Ursachen für die schlechte Fruchtbarkeit hinweist. 
Da die RFD als Maß der Körperkondition nachweislich enge Beziehungen sowohl zur Milchleistung als auch zur Fruchtbarkeit besitzt, bietet sich diese Messgröße für die Herdenüberwachung an. Das setzt allerdings die Verfügbarkeit von Referenzwerten voraus. Referenzwerte werden üblicherweise durch einen Mittelwert mit einem Streuungsmaß angegeben. Dieses Verfahren muss bei der Kondition versagen, da sich der Referenzbereich im Laktationsablauf kontinuierlich verändert. Das angewandte Regressionsmodell stellt eine elegante Lösung für dieses Problem dar (Abb. 4). Der Vorteil der dargestellten Modellierung der Referenzgrenzen liegt in der Möglichkeit der weiteren Bearbeitung verschiedener Fragestellungen einschließlich der Überprüfung der Zuverlässigkeit der vorgeschlagenen Referenzgrenzen.

$\mathrm{Zu}$ diesem Zweck wurde der Verlauf der RFD in zwei Betrieben mit annähernd gleichem Milchleistungsniveau verglichen (Abb. 5, 6). In Betrieb 1 verläuft die RFD in guter Übereinstimmung mit der Referenzkurve aus Abbildung 4. Hier sind auch die Fruchtbarkeitsergebnisse zufriedenstellend. In der anderen Herde wird die Körperkondition sehr viel stärker zur Realisierung der Milchleistung herangezogen, was in der Konsequenz zu einem Verlauf der RFD unterhalb der Referenzkurve führt (Abb. 4, 6). Die Fruchtbarkeitsergebnisse sind in dieser Herde mit einer Zwischentragezeit von 140 Tagen unbefriedigend. Milchleistung und Fruchtbarkeit werden durch die Fütterung nicht gleichmäßig gefördert und treten in eine unerwünschte Konkurrenz zueinander, was immer zu Lasten einer schlechten Fruchtbarkeit geht.

Auf Grund einer unvollständigen Datenlage konnten mögliche Zusammenhänge zwischen der Konditionsentwicklung und Erkrankungen nicht sinnvoll ausgewertet werden. An dieser Stelle ist aber darauf zu verweisen, dass die Fruchtbarkeit ein empfindlicher Parameter für eine gestörte bzw. ungestörte Tiergesundheit ist. Deshalb kann die Beziehung zwischen Milchleistung und Tiergesundheit auch durch die Betrachtung des Zusammenhanges zwischen Milchleistung und Fruchtbarkeit ersetzt werden. Der Vorzug liegt darin, dass für beide Leistungsmerkmale quantitative Maßzahlen für eine statistische Auswertung zur Verfügung stehen.

Das gesamte Untersuchungsmaterial wurde in drei Gruppen unterteilt, um zu beurteilen, wie die RFD bei einseitiger Betrachtung einer guten Fruchtbarkeit, einer hohen Milchleistung oder bei gemeinsamer Berücksichtigung einer hohen Milchleistung bei einer guten Fruchtbarkeit verläuft (Abb. 7). Die Auswertung zeigt, dass bei einseitiger Betrachtung der Fruchtbarkeit die Tiere mit einer geringen Nutzung der Körperkondition, umgekehrt bei einer einseitigen Orientierung auf eine hohe Milchleistung diejenigen mit einem intensiven Abbau der Körperkondition bevorteilt sind. Aus den bisher dargestellten Ergebnissen folgt, dass dies immer zu Lasten der anderen Leistung geht. Will man hingegen eine harmonische Abstimmung zwischen Milchleistung und Fruchtbarkeit, verläuft die RFD zwischen den beiden erstgenannten (Abb. 7). Noch wichtiger ist dabei, dass sie in der Verlaufsform der in Abbildung 4 dargestellten Referenzkurve entspricht.

Folgende Schlussfolgerungen lassen sich ableiten. Milchleistung und Fruchtbarkeit (und allgemeine Tiergesundheit) können sich sowohl synergistisch als auch antagonistisch zueinander verhalten. Dieses Verhältnis ist grundsätzlich unabhängig vom erreichten Milchleistungsniveau der Herde. Es wird vielmehr durch die äußeren Fütterungs- und Haltungsbedingungen bestimmt. Die Konditionsbeurteilung über die ultrasonographische Messung der Rückenfettdicke ist zur Beschreibung der zu erwartenden Beziehung zwischen Milchleistung und Fruchtbarkeit (und allgemeiner Gesundheit) 
geeignet. Bewegt sich die Kondition einer Herde innerhalb der vorgeschlagenen Referenzgrenzen für die RFD ist auf Basis des Energiestoffwechsels keine Konkurrenz zwischen Milchleistung und Fruchtbarkeit zu erwarten. Wird die Körperkondition über das durch die Referenzkurve vorgegebene Maß beansprucht, ist das als Signal für eintretende Konkurrenzbeziehungen zwischen Milchleistung und Fruchtbarkeit zu Lasten der letzteren zu bewerten.

\section{Literatur}

DOMEQ, J.J.; SKIDMORE, A.L.; LLOYD, J.W.; KANEENE, J.B.:

Relationship between body condition scores and milk yield in a large dairy herd of high yielding Holstein cows. J. Dairy Sci. 80 (1997a), 101-112

DOMEQ, J.J.; SKIDMORE, A.L.; LLOYD, J.W.; KANEENE, J.B.: Relationship between body condition scores and conception at artificial insemination in a large dairy herd of high yielding Holstein cows. J. Dairy Sci. 80 (1997b), 113-120

EDMONSON, A.J.; LEAN, I.J.; WEAVER, L.D.; FARVER, T.; WEBSTER, G.: A body condition scoring chart for Holstein cows. J. Dairy Sci. 72 (1989), 68-78

GALLO, L.; CARNIER, P.; CASSANDRO, M.; MANTOVANI, R.; BAILONI, L.; CONTIERO, B.; BITTANTE, G.:

Change in body condition scores of Holstein cows as affected by parity and mature equvivalent milk yield. J. Dairy Sci. 79 (1996), 1009-1015

GEARHART, M.A.; CURTIS, C.R.; ERB, H.N.; SMITH, R.D.; SNIFFEN, C.J.; CHASE, L.E.; COOPER, M.D.:

Relationship of changes in condition score to cow health in Holsteins. J. Dairy Sci. 73 (1990), 31323140

HEUER, C.; SCHUKKEN, Y.H.; DOBBELAAR, P.:

Postpartum body condition score and results from the first test day milk as predictors of disease, fertility, yield, and culling in commercial dairy herds. J. Dairy Sci. 82 (1999), 295-304

KLAWUHN, D.; STAUFENBIEL, R.:

Aussagekraft der Rückenfettdicke zum Körperfettgehalt beim Rind. Tierärztl. Praxis 25 (1997a), 133138

KLAWUHN, D.; STAUFENBIEL, R.:

Die Ermittlung der Körperzusammensetzung über die Gesamtkörperwasserbestimmung mit Phenazon zur Beschreibung des Körperfettansatzes beim Rind. Dtsch. Tierärztl. Wochenschrift 104 (1997b), 515520

LOTTHAMMER, K.H.:

Gesundheits- und Fruchtbarkeitsstörungen beim Milchrind. Tierärztlich. Praxis 9 (1981), 541-551

MARKUSFELD, O.; GALON, N; EZRA, E.:

Body condition score, health, yield and fertility in dairy cows. Vet. Rec. 141 (1997), 67-72

NEBEL, R.L.; McGILLIARD, M.L.:

Interactions of high milk yield and reproductive performance in dairy cows. J. Dairy Sci. 76 (1993), 3257-3268

PEDRON, O.; CHELLI, F; SENATORE, E.; BAROLL, D; RIZZI, R.:

Effect of body condition score at calving on performance, some blood parameters, and milk fat acid composition in dairy cows. J. Dairy Sci. 78 (1993), 2528-2535

RUEGG, P.L.; MILTON, R.L.:

Body condition scores of Holstein cows on Prince Edward Island, Canada: Relationship with yield, reproductive performance, and disease. J. Dairy Sci. 78 (1995), 552-564

STAPLES, C.R.; THATCHER, C.C.; CLARK, J.H.:

Relationship between ovarian activity and energy status during the early postpartum period of high producing dairy cows. J. Dairy Sci. 73 (1990), 938-947

STAUFENBIEL, R.; LANGHANS, J.; ROSSOW, N.; LEUTHOLD, G.:

Verhalten der Rückenfettdicke, der Aktivitäten der NADP-abhängigen Dehydrogenasen des Fettgewebes sowie der Fettgewebsbestandteile Fett und Protein und ihre Aussage zum Energiestoffwechsel der Milchkuh. Arch. exper. Vet. med. 43 (1989a), 885-895

STAUFENBIEL, R.; LANGHANS, J.; BAUER; J.; DARGEL, D.; ROSSOW, N.; LEUTHOLD, G.: Untersuchungen zur Beurteilung der postpartalen Energiebilanz der Milchkuh. Mh. Vet.-Med., 44 (1989b), 594-598 
STAUFENBIEL, R.; STAUFENBIEL, B.; LACHMANN, I.; KLUKAS, H.:

Fettstoffwechsel und Fruchtbarkeit bei der Milchkuh. Prakt. Tierarzt coll. vet. XXII (1991), 18-25

STAUFENBIEL, R.:

Energie- und Fettstoffwechsel des Rindes - Untersuchungskonzept und Messung der Rückenfettdicke. Mh. Vet.-Med. 47 (1992), 467-474

STAUFENBIEL, R.; RISCHK, U.; SCHUMACHER, B.; BECKER, W.:

Untersuchungen zum Glukose-Insulin-System der Milchkuh mittels Tagesprofilen und mit dem Glukosetoleranztest. Mh. Vet.-Med. 47 (1992a), 467-474

STAUFENBIEL, R.; MEIER, R.; HACKBARTH, K.-H.; STAUFENBIEL, B.; ROSSOW, N.:

Untersuchungen zum optimalen Fettansatz bei der Milchkuh. Mh. Vet.-Med. 47 (1992b), 125-136

STAUFENBIEL, R.; STAUFENBIEL, B.; LACHMANN, I.:

Fettstoffwechsel, Fruchtbarkeit und Gesundheit bei der Milchkuh. Arch. Tierz., Dummerstorf 36 (1993a), 121-137

STAUFENBIEL, R.; STAUFENBIEL, B.; ROSSOW, N.; KLUKAS, H.:

Energie- und Fettstoffwechsel des Rindes - Beziehungen der Rückenfettdicke zur Milchleistung, Fruchtbarkeit und zu klinisch-chemischen Parametern. Mh. Vet.-Med. 48 (1993b), 3-11

STAUFENBIEL, R.; LACHMANN, I.; BAUER, J.; STAUFENBIEL, B:

Energie- und Fettstoffwechsel des Rindes - Beziehungen der Rückenfettdicke zur Energieaufnahme und zur Energiebilanz. Mh. Vet.-Med. 48 (1993c), 58-66

STAUFENBIEL, R.:

Konditionsbeurteilung von Milchkühen mit Hilfe der sonographischen Rückenfettdickenmessung. Prakt. Tierarzt coll. vet. XXVII (1997), 87-92

VEERKAMP, R.F.; SIMM, G.; HOLDAM, J.D.:

Effects of interaction between genotype and feeding system on milk production, feed intake, efficiency and body mobilization in dairy cows. Livest. Prod. Sci. 39 (1994), 229-237

WALTNER, S.S.; McNAMARA, J.P.; HILLERS, J.K.:

Relationship of body condition score to milk production variables in high producing Holstein dairy cattle. J. Dairy Sci. 76 (1993), 3410-3419

WILDMAN, E.E.; JONES, G.M.; WAGNER, P.E.; BOMAN, R.L.; TROUTT, J.R.; LESCH, T.N.:

A dairy cow body condition scoring system and its relationship to selected production characteristics. J. Dairy Sci. 65 (1982), 495-501

Eingegangen: 27.03.2003

Akzeptiert: 23.09.2003

Autor für Korrespondenz

Prof. Dr. RUDOLF STAUFENBIEL

Klinik für Klauentiere des Fachbereiches Veterinärmedizin der Freien Universität Berlin

Königsweg 65

D-14163 Berlin 